Supporting Information

\title{
Ultrafast Protonation of an Amide: Photoionization-Induced Proton Transfer in Phenol-Dimethylformamide Complex Cation
}

\author{
Jun-Yi Wu and Po-Yuan Cheng*
}

Department of Chemistry, National Tsing Hua University, Hsinchu, Taiwan, 30043, R. O. C. 
S1. $\mathrm{PhOH}$ and $\mathrm{PhOH}-\mathrm{DMF}$ transients measured under Exc-MPI detection conditions

In order to understand the time scales of the $\mathrm{S}_{1}$ excited-state decay of the PhOH-DMF complex, we have also measured the PhOH-DMF transients under the excitation-multiphoton ionization (Exc-MPI) detection condition suitable for measuring neutral excited-state dynamics. Fig. S1 shows an Exc-MPI transient of PhOH-DMF (red circles) measured with $\lambda_{\text {pump }}=266.7$ and $\lambda_{\text {probe }}=400 \mathrm{~nm}$. This transient was recorded with very weak pump pulses $(\sim 0.3 \mu \mathrm{J} / \mathrm{pulse})$, which only allow one-photon excitation to the $\mathrm{S}_{1}$ state and produce non-detectable ion signal, and relatively intense probe pulses $(\sim 20 \mu \mathrm{J} /$ pulse $)$ to enhance the ionization of the neutral $\mathrm{S}_{1}$ state. The PI-PF detection scheme used to measure the ionic dynamics is greatly suppressed under this condition because the pump pulse alone does not produce $[\mathrm{PhOH}-\mathrm{DMF}]^{+}$ions. ${ }^{1}$ As shown in Fig. $\mathrm{S} 1$, the PhOH-DMF transient thus obtained exhibits a very long decay of about $2.5 \mathrm{~ns}$. Also shown in Fig. $\mathrm{S} 1$ is a $\mathrm{PhOH}$ monomer transient (blue circles) measured under the same condition, which gives a decay of about $1.5 \mathrm{~ns}$, consistent with the phenol $\mathrm{S}_{1}$-state lifetime in this energy region ( $1200 \mathrm{~cm}^{-1}$ above $\mathrm{S}_{1}$ origin). ${ }^{2}$ Thus, hydrogen-bonding of phenol with DMF seems to slightly lengthen its $\mathrm{S}_{1}$-state lifetime. Moreover, this result confirms that the temporal components ( $25 \mathrm{ps})$ observed in the PI-PF ion transients shown in Figure 4 of the main text cannot be due to the much slower $\mathrm{S}_{1}$-state dynamics of the neutral $\mathrm{PhOH}-\mathrm{DMF}$ complex.

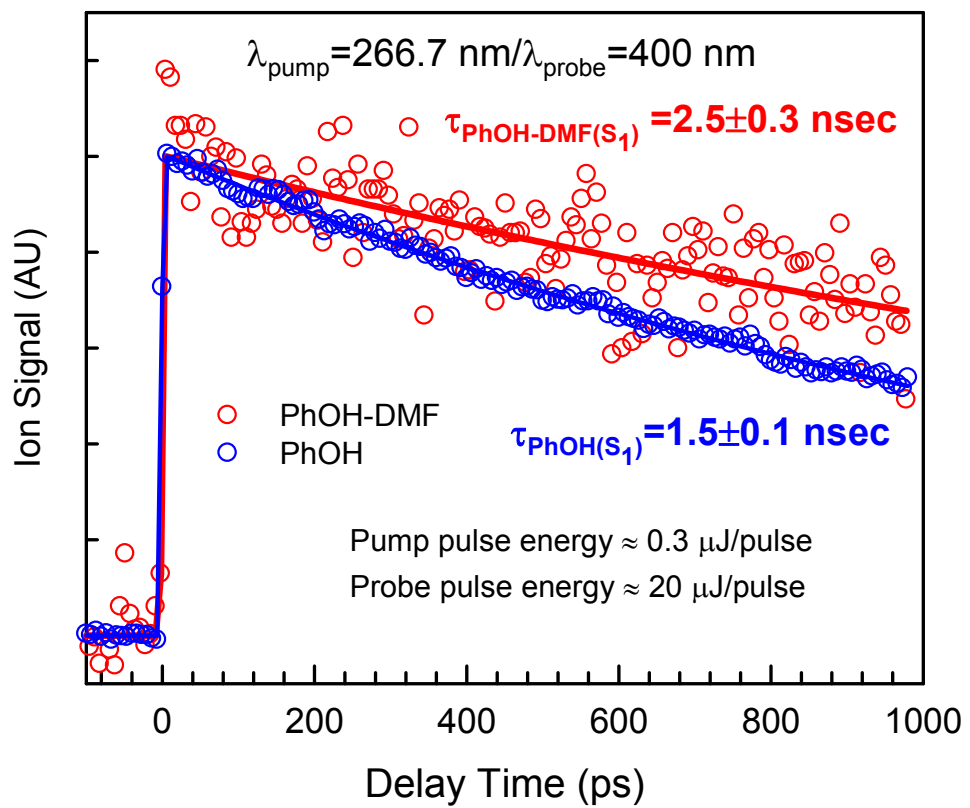

Fig. S1 PhOH-DMF (red open circles) and $\mathrm{PhOH}$ (blue open circles) transients measured under Exc-MPI detection conditions with $\lambda_{\text {pump }}=266.7$ and $\lambda_{\text {probe }}=400 \mathrm{~nm}$. The pump pulse energy was reduced to such a low level ( $\sim 0.3 \mu \mathrm{J} /$ pulse) that no ion signal was detected by the pump laser alone, while the probe pulse energy was raised $(\sim 20 \mu \mathrm{J} /$ pulse) to enhance the two-photon ionization efficiency. The solid lines are the best fit of the data to a single exponential decay convoluted with a Gaussian instrument response function. 
S2. Neutral PhOH-DMF ground-state optimized structures calculated at $\omega B 97 X D /$ aug-cc-pVDZ

(1) neutral $\mathrm{PhOH}-\mathrm{DMF} \mathrm{O} \mathrm{O}_{\mathrm{A}}$-bound isomer ( $\mathrm{A}$ isomer)

Cartesian coordinates

$\mathrm{X}$

$\mathrm{Y}$

Z

$\begin{array}{lrrr}\mathrm{C} & 2.3076970000 & 0.6982850000 & 0.7857620000 \\ \mathrm{C} & 3.0091770000 & -0.4986400000 & 0.6722720000 \\ \mathrm{C} & 2.6646750000 & -1.4385700000 & -0.3012830000 \\ \mathrm{C} & 1.6173090000 & -1.1543140000 & -1.1768760000 \\ \mathrm{C} & 0.9139920000 & 0.0454890000 & -1.0808530000 \\ \mathrm{C} & 1.2464620000 & 0.9721810000 & -0.0842850000 \\ \mathrm{H} & 2.5618550000 & 1.4322300000 & 1.5496400000 \\ \mathrm{H} & 3.8332410000 & -0.7019720000 & 1.3570350000 \\ \mathrm{H} & 3.2151820000 & -2.3750370000 & -0.3837600000 \\ \mathrm{H} & 1.3498660000 & -1.8690350000 & -1.9562690000 \\ \mathrm{H} & 0.1130740000 & 0.2763660000 & -1.7820230000 \\ \mathrm{O} & 0.5765760000 & 2.1370680000 & 0.0800330000 \\ \mathrm{H} & -0.3162160000 & 2.0629650000 & -0.3076900000 \\ \mathrm{~N} & -2.2150850000 & -0.4919450000 & 0.3398360000 \\ \mathrm{C} & -2.4440870000 & 0.3974200000 & -0.6424630000 \\ \mathrm{H} & -3.0849950000 & -0.0075540000 & -1.4486190000 \\ \mathrm{O} & -2.0208520000 & 1.5498210000 & -0.6907300000 \\ \mathrm{C} & -1.4248070000 & -0.1540380000 & 1.5100230000 \\ \mathrm{H} & -0.4549120000 & -0.6700320000 & 1.4807660000 \\ \mathrm{H} & -1.2537840000 & 0.9245530000 & 1.5329580000 \\ \mathrm{H} & -1.9694150000 & -0.4586670000 & 2.4138570000 \\ \mathrm{C} & -2.6587940000 & -1.8657690000 & 0.2274340000 \\ \mathrm{H} & -1.7935740000 & -2.5435750000 & 0.1872830000 \\ \mathrm{H} & -3.2806340000 & -2.1392080000 & 1.0914170000 \\ \mathrm{H} & -3.2496280000 & -1.9947850000 & -0.6862640000\end{array}$

(2) neutral PhOH-DMF $\mathrm{O}_{\mathrm{B}}$-bound isomer (B isomer)

Cartesian coordinates

$\mathrm{X}$

$\mathrm{Y}$

Z

$\begin{array}{llll}\mathrm{C} & -1.4433240000 & 0.2061300000 & -0.8867870000 \\ \mathrm{C} & -2.3343660000 & 1.2706900000 & -1.0213530000 \\ \mathrm{C} & -3.4876610000 & 1.3398080000 & -0.2419850000\end{array}$ 


$\begin{array}{lrrr}\mathrm{C} & -3.7449270000 & 0.3225290000 & 0.6797170000 \\ \mathrm{C} & -2.8634270000 & -0.7434700000 & 0.8283280000 \\ \mathrm{C} & -1.7033360000 & -0.8061330000 & 0.0467920000 \\ \mathrm{H} & -0.5530640000 & 0.1458680000 & -1.5129170000 \\ \mathrm{H} & -2.1243430000 & 2.0512250000 & -1.7538040000 \\ \mathrm{H} & -4.1816300000 & 2.1720990000 & -0.3544010000 \\ \mathrm{H} & -4.6460630000 & 0.3594670000 & 1.2930910000 \\ \mathrm{H} & -3.0564230000 & -1.5410740000 & 1.5449570000 \\ \mathrm{O} & -0.8774970000 & -1.8618530000 & 0.2312330000 \\ \mathrm{H} & -0.0401360000 & -1.7311730000 & -0.2581890000 \\ \mathrm{C} & 1.8889550000 & -0.3070000000 & 0.1421670000 \\ \mathrm{O} & 1.6113630000 & -1.1761280000 & -0.6825940000 \\ \mathrm{~N} & 3.0717750000 & 0.3271800000 & 0.2265470000 \\ \mathrm{H} & 1.1535760000 & 0.0261110000 & 0.8982100000 \\ \mathrm{C} & 4.1518640000 & 0.0129030000 & -0.6884540000 \\ \mathrm{H} & 5.0343630000 & -0.3259330000 & -0.1275830000 \\ \mathrm{H} & 3.8208000000 & -0.7799180000 & -1.3639590000 \\ \mathrm{H} & 4.4249740000 & 0.9034470000 & -1.2724360000 \\ \mathrm{C} & 3.3138900000 & 1.3504370000 & 1.2218680000 \\ \mathrm{H} & 3.5592370000 & 2.3070500000 & 0.7383330000 \\ \mathrm{H} & 2.4184460000 & 1.4884520000 & 1.8377530000 \\ \mathrm{H} & 4.1508920000 & 1.0626140000 & 1.8742390000\end{array}$

(3) TS structure between neutral PhOH-DMF $\mathbf{A}$ and $\mathbf{B}$ isomers Cartesian coordinates

$\mathrm{X}$ $\mathrm{Y}$ Z

$\begin{array}{lrrr}\mathrm{C} & 1.5292030000 & -0.0267420000 & 1.0344680000 \\ \mathrm{C} & 2.4990230000 & 0.9745850000 & 1.0730340000 \\ \mathrm{C} & 3.3922250000 & 1.1439020000 & 0.0162960000 \\ \mathrm{C} & 3.3075810000 & 0.2924330000 & -1.0871910000 \\ \mathrm{C} & 2.3406070000 & -0.7068220000 & -1.1429980000 \\ \mathrm{C} & 1.4430620000 & -0.8687060000 & -0.0818520000 \\ \mathrm{H} & 0.8456470000 & -0.1737720000 & 1.8712290000 \\ \mathrm{H} & 2.5614800000 & 1.6226050000 & 1.9481850000 \\ \mathrm{H} & 4.1510570000 & 1.9247070000 & 0.0541630000 \\ \mathrm{H} & 4.0041010000 & 0.4082680000 & -1.9184380000 \\ \mathrm{H} & 2.2662140000 & -1.3745690000 & -2.0006030000\end{array}$




$\begin{array}{lrrr}\mathrm{O} & 0.5236400000 & -1.8598890000 & -0.1782010000 \\ \mathrm{H} & -0.1688640000 & -1.7276100000 & 0.4912660000 \\ \mathrm{~N} & -2.7855780000 & 0.4641360000 & -0.1383640000 \\ \mathrm{C} & -1.8508740000 & 0.2020250000 & 0.7927880000 \\ \mathrm{H} & -1.2546350000 & 1.0909120000 & 1.0731640000 \\ \mathrm{O} & -1.6437640000 & -0.8986240000 & 1.2984980000 \\ \mathrm{C} & -3.6370490000 & -0.5902580000 & -0.6540010000 \\ \mathrm{H} & -3.4425670000 & -0.7451440000 & -1.7249020000 \\ \mathrm{H} & -3.4226250000 & -1.5127020000 & -0.1084790000 \\ \mathrm{H} & -4.6935880000 & -0.3189630000 & -0.5204520000 \\ \mathrm{C} & -2.9351460000 & 1.7846280000 & -0.7123030000 \\ \mathrm{H} & -3.9546950000 & 2.1621820000 & -0.5489240000 \\ \mathrm{H} & -2.2239240000 & 2.4749960000 & -0.2454810000 \\ \mathrm{H} & -2.7393490000 & 1.7579690000 & -1.7940080000\end{array}$

(4) neutral PhOH-DMF N-bound isomer ( $\mathbf{N}$ isomer)

Cartesian coordinates $\mathrm{X}$ $\mathrm{Y}$

$\mathrm{Z}$

$\begin{array}{lrrr}\mathrm{C} & 2.4492190000 & -1.3152260000 & -0.1462650000 \\ \mathrm{C} & 3.5842360000 & -0.5738840000 & 0.1699830000 \\ \mathrm{C} & 3.5185040000 & 0.8157230000 & 0.2847640000 \\ \mathrm{C} & 2.3004610000 & 1.4599530000 & 0.0751110000 \\ \mathrm{C} & 1.1552560000 & 0.7317700000 & -0.2453090000 \\ \mathrm{C} & 1.2340310000 & -0.6591860000 & -0.3519860000 \\ \mathrm{H} & 2.4889420000 & -2.3998170000 & -0.2390050000 \\ \mathrm{H} & 4.5317490000 & -1.0895930000 & 0.3288430000 \\ \mathrm{H} & 4.4099600000 & 1.3906120000 & 0.5328440000 \\ \mathrm{H} & 2.2340580000 & 2.5450200000 & 0.1558240000 \\ \mathrm{H} & 0.2086730000 & 1.2483370000 & -0.4174260000 \\ \mathrm{O} & 0.1486000000 & -1.4280990000 & -0.6599960000 \\ \mathrm{H} & -0.6366820000 & -0.8673540000 & -0.6876300000 \\ \mathrm{~N} & -2.4701850000 & -0.1645340000 & 0.2102890000 \\ \mathrm{C} & -2.6533800000 & 0.8802240000 & -0.6498670000 \\ \mathrm{H} & -3.2342720000 & 0.5929600000 & -1.5498600000 \\ \mathrm{O} & -2.2070490000 & 2.0014220000 & -0.4952690000 \\ \mathrm{C} & -1.8734270000 & 0.0643360000 & 1.5176420000 \\ \mathrm{H} & -1.1533870000 & -0.7335470000 & 1.7396810000\end{array}$




$\begin{array}{lrrr}\mathrm{H} & -1.3598970000 & 1.0287910000 & 1.5082200000 \\ \mathrm{H} & -2.6504630000 & 0.0750400000 & 2.2964700000 \\ \mathrm{C} & -3.2007520000 & -1.4073770000 & 0.0361020000 \\ \mathrm{H} & -2.5307160000 & -2.2610820000 & 0.2029560000 \\ \mathrm{H} & -4.0410560000 & -1.4749070000 & 0.7438420000 \\ \mathrm{H} & -3.5929080000 & -1.4673070000 & -0.9857140000\end{array}$

(5) TS structure between neutral PhOH-DMF $\mathbf{N}$ and $\mathbf{A}$ isomers

$\begin{array}{lccc} & \text { Cartesian coordinates } & \\ & \mathrm{X} & \mathrm{Y} & \mathrm{Z} \\ \mathrm{C} & 2.3583480000 & -1.3336240000 & -0.2227240000 \\ \mathrm{C} & 3.5021710000 & -0.7000680000 & 0.2557690000 \\ \mathrm{C} & 3.5214210000 & 0.6815610000 & 0.4527260000 \\ \mathrm{C} & 2.3800590000 & 1.4280030000 & 0.1635600000 \\ \mathrm{C} & 1.2269350000 & 0.8091740000 & -0.3174790000 \\ \mathrm{C} & 1.2223530000 & -0.5745700000 & -0.5076960000 \\ \mathrm{H} & 2.3324530000 & -2.4106820000 & -0.3833980000 \\ \mathrm{H} & 4.3893120000 & -1.2944770000 & 0.4761680000 \\ \mathrm{H} & 4.4194770000 & 1.1716370000 & 0.8269880000 \\ \mathrm{H} & 2.3813840000 & 2.5082080000 & 0.3103200000 \\ \mathrm{H} & 0.3335940000 & 1.3972600000 & -0.5396460000 \\ \mathrm{O} & 0.1220440000 & -1.2407500000 & -0.9705920000 \\ \mathrm{H} & -0.5951460000 & -0.6111980000 & -1.0933210000 \\ \mathrm{~N} & -2.5545810000 & -0.1367130000 & 0.3306540000 \\ \mathrm{C} & -2.7117940000 & 0.8513920000 & -0.5861100000 \\ \mathrm{H} & -3.4507810000 & 0.5897210000 & -1.3707720000 \\ \mathrm{O} & -2.1081130000 & 1.9122700000 & -0.5934150000 \\ \mathrm{C} & -1.7042570000 & 0.0488620000 & 1.4930120000 \\ \mathrm{H} & -0.9383480000 & -0.7371840000 & 1.5310280000 \\ \mathrm{H} & -1.2196340000 & 1.0257340000 & 1.4225480000 \\ \mathrm{H} & -2.3089700000 & 0.0048980000 & 2.4103290000 \\ \mathrm{C} & -3.3060900000 & -1.3721720000 & 0.2543060000 \\ \mathrm{H} & -3.9735460000 & -1.4763040000 & 1.1225780000 \\ \mathrm{H} & -3.9128660000 & -1.3826480000 & -0.6584880000 \\ & -2.6211770000 & -2.2314860000 & 0.2309590000\end{array}$



S3. $[\mathrm{PhOH}-\mathrm{DMF}]^{+}$cation ground-state optimized structures calculated at
$\omega \mathrm{B} 97 \mathrm{XD} / \mathrm{aug}-\mathrm{cc}-\mathrm{p} V \mathrm{DZ}$

(1) $[\mathrm{PhOH}-\mathrm{DMF}]^{+}$cation $\mathbf{A}_{\mathrm{NPT}}^{+}$isomer

Cartesian coordinates

$\mathrm{X}$

$\mathrm{Y}$

Z

C $\quad-2.6406430000$

1.3513850000

$-0.0000830000$

C $\quad-3.9305890000$

0.8918290000

$-0.0000050000$

C $\quad-4.1829720000$

$-0.5011280000$

0.0000930000

$\mathrm{C}$

$-3.1213800000$

$-1.4391390000$

0.0001090000

C $\quad-1.8203560000$

$-1.0105240000$

0.0000320000

C $\quad-1.5540150000$

0.4063190000

$-0.0000640000$

$\mathrm{H} \quad-2.3957660000$

2.4121860000

$-0.0001600000$

$\mathrm{H} \quad-4.7651940000$

1.5909370000

$-0.0000170000$

$\mathrm{H} \quad-5.2123510000$

$-0.8588820000$

0.0001560000

H $\quad-3.3473450000$

$-2.5042260000$

0.0001840000

$\mathrm{H} \quad-0.9790370000$

$-1.7020610000$

0.0000400000

$\mathrm{O}$

$-0.3588630000$

0.8794300000

$-0.0001350000$

$\mathrm{H}$

0.4063640000

0.1450400000

$-0.0001170000$

$\mathrm{N}$

3.3905030000

$-0.0027410000$

0.0000330000

C

2.5480260000

$-1.0215330000$

$-0.0000950000$

$\mathrm{H} \quad 3.0102520000$

$-2.0177420000$

$-0.0001930000$

$\mathrm{O}$

1.3009580000

$-0.9310950000$

$-0.0001120000$

C

2.8856050000

1.3666000000

0.0001870000

$\mathrm{H} \quad 2.2795780000$

1.5525740000

$-0.8953110000$

$\mathrm{H} \quad 2.2791940000$

1.5522210000

0.8954950000

$\mathrm{H}$

3.7354160000

2.0535150000

0.0005060000

$\mathrm{C}$

4.8346220000

$-0.2102640000$

0.0000150000

$\mathrm{H} \quad 5.2767650000$

0.2450990000

$-0.8945700000$

$\mathrm{H} \quad 5.2767580000$

0.2448870000

0.8947110000

$\mathrm{H}$

5.0553030000

$-1.2823010000$

$-0.0001120000$

(2) $[\mathrm{PhOH}-\mathrm{DMF}]^{+}$cation $\mathbf{A}_{\mathrm{PT}}^{+}$isomer

Cartesian coordinates

$\mathrm{X}$

$\begin{array}{ll}\mathrm{C} & 2.4880330000 \\ \mathrm{C} & 3.8217540000\end{array}$
$\mathrm{Y}$

1.3595370000

1.0397210000
Z

0.0000860000

0.0001820000 


$\begin{array}{lrrr}\mathrm{C} & 4.2234740000 & -0.3145540000 & 0.0001400000 \\ \mathrm{C} & 3.2687360000 & -1.3579960000 & 0.0000000000 \\ \mathrm{C} & 1.9286470000 & -1.0682380000 & -0.0000980000 \\ \mathrm{C} & 1.4913020000 & 0.3114830000 & -0.0000560000 \\ \mathrm{H} & 2.1376510000 & 2.3905860000 & 0.0001150000 \\ \mathrm{H} & 4.5762470000 & 1.8250120000 & 0.0002910000 \\ \mathrm{H} & 5.2850820000 & -0.5600530000 & 0.0002160000 \\ \mathrm{H} & 3.6065130000 & -2.3933530000 & -0.0000310000 \\ \mathrm{H} & 1.1762950000 & -1.8558140000 & -0.0002080000 \\ \mathrm{O} & 0.2632530000 & 0.6296240000 & -0.0001390000 \\ \mathrm{H} & -0.6959610000 & -0.3210160000 & -0.0002110000 \\ \mathrm{~N} & -3.3375550000 & 0.0510290000 & -0.0000010000 \\ \mathrm{C} & -2.6469030000 & -1.0583230000 & -0.0000040000 \\ \mathrm{H} & -3.2093580000 & -1.9952490000 & 0.0001900000 \\ \mathrm{O} & -1.3800560000 & -1.1696980000 & -0.0002150000 \\ \mathrm{C} & -2.6873800000 & 1.3647830000 & -0.0003220000 \\ \mathrm{H} & -2.0638830000 & 1.4799640000 & -0.8938890000 \\ \mathrm{H} & -3.4648560000 & 2.1316640000 & -0.0009980000 \\ \mathrm{H} & -2.0646780000 & 1.4807320000 & 0.8937210000 \\ \mathrm{C} & -4.8013640000 & 0.0155660000 & 0.0004180000 \\ \mathrm{H} & -5.1757370000 & 0.5250900000 & 0.8957690000 \\ \mathrm{H} & -5.1762500000 & 0.5247920000 & -0.8948870000 \\ \mathrm{H} & -5.1515360000 & -1.0208460000 & 0.0006950000\end{array}$

(3) $[\mathrm{PhOH}-\mathrm{DMF}]^{+}$cation $\mathbf{B}^{+}$isomer

Cartesian coordinates

$\mathrm{X}$

$\begin{array}{ll}\text { C } & -2.0703630000 \\ \text { C } & -3.3680980000 \\ \text { C } & -4.4355850000 \\ \text { C } & -4.1910420000 \\ \text { C } & -2.9023870000 \\ \text { C } & -1.7910380000 \\ \text { H } & -1.2322290000 \\ \text { H } & -3.5854970000 \\ \text { H } & -5.4621820000 \\ \text { H } & -5.0304180000 \\ \text { H } & -2.6723490000\end{array}$

Y

1.0133520000

1.4555840000

0.5277690000

$-0.8629260000$

$-1.3339260000$

$-0.4071870000$

1.7095900000

2.5227540000

0.8929450000

$-1.5568090000$

$-2.3983300000$
Z

$-0.0000150000$

$-0.0000180000$

$-0.0000040000$

0.0000110000

0.0000140000

0.0000020000

$-0.0000290000$

$-0.0000310000$

$-0.0000050000$

0.0000200000

0.0000250000 


$\begin{array}{lrrr}\mathrm{O} & -0.6084580000 & -0.8584660000 & 0.0000060000 \\ \mathrm{H} & 0.5227330000 & -0.0574420000 & 0.0000000000 \\ \mathrm{C} & 2.4300660000 & -0.3927140000 & -0.0000040000 \\ \mathrm{O} & 1.4644560000 & 0.4423130000 & 0.0000040000 \\ \mathrm{~N} & 3.6788700000 & -0.0186790000 & 0.0000080000 \\ \mathrm{H} & 2.2193360000 & -1.4670240000 & -0.0000240000 \\ \mathrm{C} & 4.0689980000 & 1.3908110000 & 0.0000270000 \\ \mathrm{H} & 4.6705400000 & 1.5921390000 & -0.8942330000 \\ \mathrm{H} & 3.1796070000 & 2.0225020000 & 0.0002300000 \\ \mathrm{H} & 4.6708560000 & 1.5920130000 & 0.8941020000 \\ \mathrm{C} & 4.7612170000 & -1.0009530000 & -0.0000270000 \\ \mathrm{H} & 5.3792390000 & -0.8582220000 & 0.8942510000 \\ \mathrm{H} & 4.3504060000 & -2.0148680000 & -0.0000870000 \\ \mathrm{H} & 5.3792650000 & -0.8581270000 & -0.8942710000\end{array}$

(4) TS structure between $[\mathrm{PhOH}-\mathrm{DMF}]^{+}$cation $\mathbf{A}^{+}$and $\mathbf{B}^{+}$isomers

\begin{tabular}{lccc} 
& \multicolumn{2}{c}{ Cartesian coordinates } \\
& $\mathrm{X}$ & $\mathrm{Y}$ & $\mathrm{Z}$ \\
$\mathrm{C}$ & 2.9329790000 & -1.2889200000 & -0.2712230000 \\
$\mathrm{C}$ & 4.1472500000 & -0.6911750000 & -0.0634470000 \\
$\mathrm{C}$ & 4.2105700000 & 0.6906630000 & 0.2388300000 \\
$\mathrm{C}$ & 3.0353720000 & 1.4774770000 & 0.3317940000 \\
$\mathrm{C}$ & 1.8060420000 & 0.9088020000 & 0.1290840000 \\
$\mathrm{C}$ & 1.7352490000 & -0.4966230000 & -0.1779460000 \\
$\mathrm{H}$ & 2.8302370000 & -2.3470460000 & -0.5062440000 \\
$\mathrm{H}$ & 5.0660620000 & -1.2714500000 & -0.1289820000 \\
$\mathrm{H}$ & 5.1809800000 & 1.1588130000 & 0.4026220000 \\
$\mathrm{H}$ & 3.1175020000 & 2.5377610000 & 0.5652880000 \\
$\mathrm{H}$ & 0.8796840000 & 1.4784400000 & 0.1893260000 \\
$\mathrm{O}$ & 0.6152480000 & -1.0998040000 & -0.3786150000 \\
$\mathrm{H}$ & -0.2042800000 & -0.4836200000 & -0.3008020000 \\
$\mathrm{~N}$ & -3.5529750000 & 0.0635120000 & 0.0405420000 \\
$\mathrm{C}$ & -2.4869920000 & 0.5766310000 & -0.5539980000 \\
$\mathrm{H}$ & -2.7068780000 & 1.1597010000 & -1.4619310000 \\
$\mathrm{O}$ & -1.3127250000 & 0.4605950000 & -0.1461600000 \\
$\mathrm{C}$ & -3.4451600000 & -0.7267420000 & 1.2587890000 \\
$\mathrm{H}$ & -2.4110600000 & -0.7133460000 & 1.6092250000 \\
$\mathrm{H}$ & -4.1000090000 & -0.2980340000 & 2.0274400000
\end{tabular}




$\begin{array}{lrrr}\mathrm{H} & -3.7578010000 & -1.7601190000 & 1.0602450000 \\ \mathrm{C} & -4.8916590000 & 0.2621240000 & -0.4981730000 \\ \mathrm{H} & -5.3477880000 & -0.7092320000 & -0.7276480000 \\ \mathrm{H} & -5.5154730000 & 0.7847030000 & 0.2379400000 \\ \mathrm{H} & -4.8424390000 & 0.8590960000 & -1.4143390000\end{array}$

\section{S4. Isomerization between neutral $\mathrm{N}$-bound and $\mathrm{O}_{\mathrm{A}}$-bound $\mathrm{PhOH}-\mathrm{DMF}$ complex as calculated at $\omega B 97 X D /$ aug-cc-pVDZ}

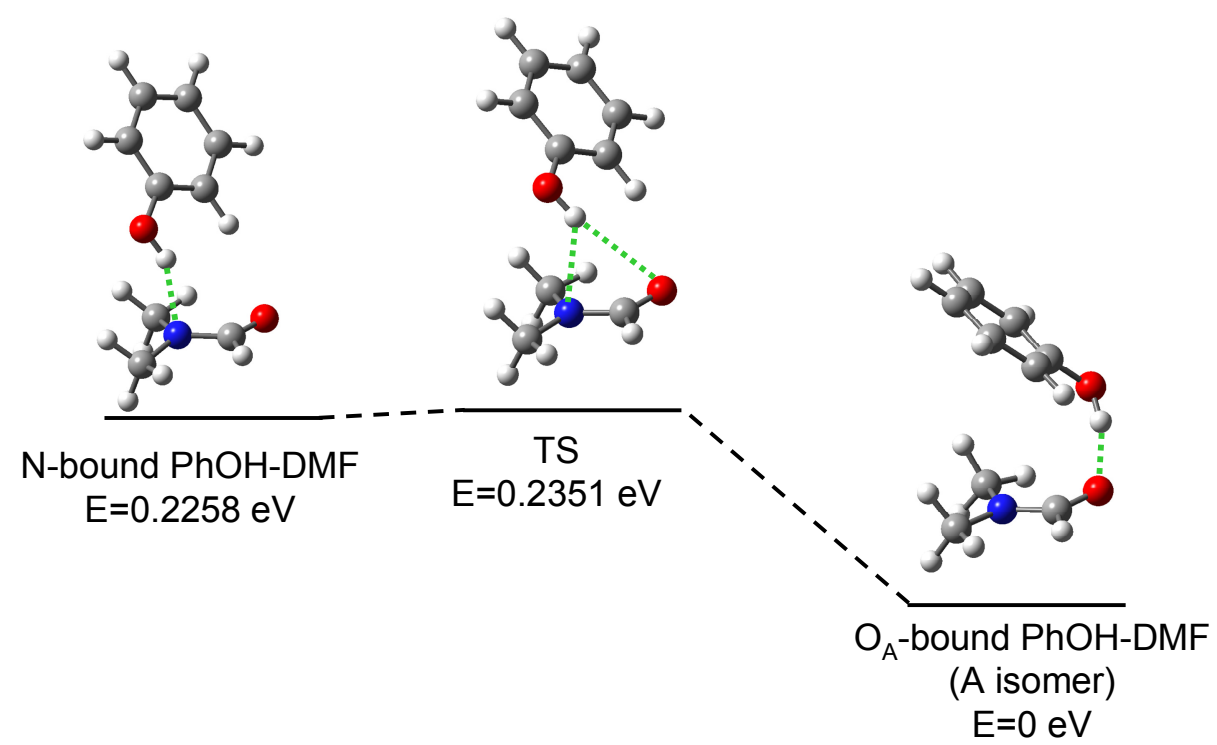

Figure S2. A schematic energy level diagram based on the DFT calculations at $\omega B 97 X D / a u g-c c-p V D Z$ level of theory for isomerization between the neutral N-bound and $\mathrm{O}_{\mathrm{A}}$-bound $\mathrm{PhOH}-\mathrm{DMF}$ complexes. The optimized structures and relative energy (not ZPE corrected) are also given. The TS structure is found to locate at about $0.0093 \mathrm{eV}\left(\sim 75 \mathrm{~cm}^{-1}\right)$ above the N-bound $\mathrm{PhOH}-\mathrm{DMF}$ complex, indicating that structural relaxation from N-bound to O-bound structures is facile during collisional cooling in supersonic expansion.

\section{Reference}

(1) Shen, C. C.; Tsai, T. T.; Wu, J. Y.; Ho, J. W.; Chen, Y. W.; Cheng, P. Y. Watching Proton Transfer in Real Time: Ultrafast Photoionization-Induced Proton Transfer in Phenol-Ammonia Complex Cation. J. Chem. Phys. 2017, 147, 164302.

(2) Lai, H. Y.; Jhang, W. R.; Tseng, C. M. Communication: Mode-Dependent Excited-State Lifetime of Phenol under the $\mathrm{S}_{1} / \mathrm{S}_{2}$ Conical Intersection. J. Chem. Phys. 2018, 149. 